\title{
Interculturalidad y Comunitarismo en la Educación Superior: la experiencia de Bluefields Indian \& Caribbean University
}

\section{Interculturality and Communitarianism in Higher Education: the Bluefields Indian \& Caribbean University experience}

\author{
Rene Alfonso Cassells ${ }^{1}$ \\ Bluefields Indian \& Caribbean University \\ Ramón A. Parajón ${ }^{2}$ \\ Universidad Nacional Autónoma de Nicaragua
}

\begin{abstract}
RESUMEN
La calidad de la educación superior siempre ha sido un tema polémico al momento de su discusión; si a esta realidad sumamos la integración de la interculturalidad y comunitarismo como elementos esenciales en la formación del nuevo profesional, la tradicional formación técnica trasciende hacia una visión holística. A partir de esta realidad nace lo que hoy conocemos como universidades comunitarias con enfoque intercultural. Nicaragua no es la excepción, dada la presencia de las universidades BICU y URACCAN, ambos casos interesantes de estudiar. Es a partir de esta experiencia que se ha elaborado este artículo de revisión en el cual se abordan los orígenes de este tipo de universidades, así como la base legal local, regional, nacional e internacional que sostienen la necesidad no solo de su creación sino de su consolidación y expansión. Con ello se posibilita la presentación de una conceptualización integradora y amplia de lo que es una Universidad Comunitaria e Intercultural que atiende las necesidades de formación técnica y profesional de sus estudiantes, docentes y población, sin obviar la calidad de la educación integradora de los saberes y conocimientos ancestrales de los pueblos históricamente marginados.

Palabras claves: Universidad, interculturalidad, minorías étnicas, pertinencia académica, inclusividad, Nicaragua-Costa Caribe.
\end{abstract}

Recibido: 16 de febrero del 2019

Aceptado: 24 de abril del 2019

\footnotetext{
${ }^{1}$ Ph.D. en Gestión de la Calidad de la Investigación Científica, por FAREM-ESTELI, UNAN-MANAGUA, Nicaragua (2009) guabul59@gmail.com

${ }^{2}$ Ph.D. de Ciencias Matemáticas, por Universidad de Valencia, España (2001) ramon.a.parajon@uv.es
} 


\begin{abstract}
The quality of higher education has always been a controversial issue at the time of its discussion, if to this reality we add the integration of interculturalism and communitarianism as essential elements in the formation of the new professional, transcending the traditional technical education through a holistic vision. From this reality, what we know today as community universities with an intercultural approach is born. Nicaragua is not the exception with the presence of the BICU and URACCAN universities, both interesting cases of studying. It is from this experience that this review paper has been prepared in which the origins of this type of universities are addressed, the local, regional, national and international legal base that supports the need not only for its creation but also for its consolidation and expansion. This allows the presentation of an integrating and comprehensive conceptualization of what is a Community and Intercultural University that meets the needs of technical and professional training of its students, teachers and population without neglecting the quality of education that integrates the knowledge and ancestral knowledge of historically marginalized peoples.
\end{abstract}

Keywords: Academic quality, inclusiveness, educational relevance, ethnic minorities.

\title{
INTRODUCCIÓN
}

Los resultados de esta investigación están referidos al modelo educativo y organizacional de Bluefields Indian \& Caribbean University (BICU) desde la perspectiva comunitaria e intercultural. La propuesta que se plantea no es una receta o panacea, sino un documento orientador que en todo caso requiere la apropiación, compromiso, voluntad política institucional y una gestión participativa y efectiva de todos los actores en la BICU. Esto, con el objetivo de aportar a la transformación estructural, educativa y académica propia de una universidad verdaderamente comunitaria e intercultural, con identidad y compromiso social para con los pueblos y comunidades, históricamente marginados, de la Costa Caribe.

Por ello, en la presente investigación se responde a la interrogante acerca de ¿cuál es, en la Bluefields Indian \& Caribbean University, el estado del arte desde la perspectiva comunitaria e intercultural? ¿Cuáles son las características que definen las diferencias de una universidad pública respecto a otra de carácter comunitario e intercultural? De allí que el objetivo de este escrito sea determinar el estado del arte de las universidades comunitarias e interculturales.

\section{EL ESTADO DEL ARTE}

\section{Aspectos históricos y culturales de la Costa Caribe}

Desde mediados del siglo XVI hasta la fecha, la Costa Caribe, por razones religiosas, comerciales, enclaves mineros, madereros y pesqueros, ha sido poblada principalmente por migrantes europeos, chinos, alemanes, canadienses y norteamericanos, generando así una gran diversidad cultural. Antes de la llegada de los europeos, la distribución regional de los pueblos indígenas y grupos étnicos se dividía en tres núcleos poblacionales (litoral, ribereña 
y del interior). Lo anterior se basaba en la relación existente entre la dispersión geográfica de las unidades familiares y el poco intercambio interregional de productos alimenticios.

La anexión forzada del Reino de la Moskitia al resto de Nicaragua, en 1912, y la economía de enclave produjeron cambios socioculturales en la población nativa local. Hasta el año de 1979, a excepción de las poblaciones mestizas, los pueblos indígenas y comunidades étnicas de la costa caribe nicaragüense, se mantuvieron de manera estable en sus territorios geográficos. A raíz de los conflictos bélicos vividos entre 1980 y 1990, se acrecentaron los flujos migratorios a lo interno del Caribe en Nicaragua. Los pueblos indígenas, social y culturalmente afectados, fueron los miskitos de la Cuenca de la Desembocadura del Río Grande de Matagalpa, y los mayangnas, errónea y despectivamente llamados sumos, de la comunidad de Musawás en el Atlántico Norte y de Karawala en el Atlántico Sur (Gonzáles, 1997).

La coexistencia de los pueblos indígenas miskitos. ramas, mayangnas y grupos étnicos creoles, garífunas y mestizos, aunada al vaivén migratorio en la ciudad de Bluefields, genera una constante riqueza cultural e importante reto socio cultural para la organización del Estado nicaragüense y en especial para la Costa Caribe.

La ciudad de Bluefields es la principal cabecera de la Región Autónoma Costa Caribe Sur (RACCS). Actualmente, en Bluefields la población mestiza es la etnia mayoritaria seguida de los creoles, con un 55\% y 35\%, respectivamente (INIDE, 2012). La densidad poblacional, según las cifras oficiales de la municipalidad de Bluefields, es de $8.9 \mathrm{hab} / \mathrm{km}^{2}$, una de la más bajas a nivel de los municipios de Nicaragua. La superficie del municipio es de $4,774.75 \mathrm{~km}^{2}$, con una población aproximada de ochenta mil habitantes. El Caribe nicaragüense representa aproximadamente dos tercios del territorio nacional y está organizado en dos regiones autónomas, RACCN y RACCS, en las cuales habita aproximadamente el $15 \%$ de la población nacional, parte de la cual es singular por su historia y diversidad étnica.

Esta diferenciación étnica se ha expresado en una histórica demanda por el reconocimiento y respeto a su condición de comunidad con características y organización social culturalmente diferentes, pero marginadas y rezagadas en cuanto al acceso a bienes y servicios básicos, infraestructura, salud y, especialmente, en el área de educación técnica y superior. Para muchos ciudadanos de la Costa Caribe, el rezago social y educativo inició durante el régimen del presidente José Santos Zelaya, cuando el acceso a la educación en lengua materna, de la cual gozaban los estos ciudadanos, fue suprimida. Esto significó una gran pérdida de identidad para las familias de la época y un retraso para el desarrollo de esta región. 


\section{El Programa de Educación Bilingüe y su espectro jurídico en la Costa Caribe de Nicaragua}

La primera institución educativa formal en la Costa Caribe, en especial Bluefields que practicaba el bilingüismo (español e inglés) en la aulas de clases, fue la Iglesia Morava con más de 165 años de presencia ininterrumpida en la región, seguida de la Iglesia Católica: jesuitas y capuchinos en la década de los 1950. Inicialmente, la presencia de éstas fue en misión evangelizadora por la cual lograron fundar, con mucho suceso y alto prestigio, carreras técnicas de contabilidad en el Colegio Moravo y el Cristóbal Colón, respectivamente; al extremo que desde la capital Managua y otras regiones del país, grandes y prestigiosas empresas públicas y privadas venían a la ciudad de Bluefields a contratar técnicos recién graduados en contabilidad. En cuanto a educación superior, en la década de los 1970 hubo esfuerzos de la Universidad Nacional Autónoma de Nicaragua para fundar un recinto en la ciudad de Bluefields, fallando en sus dos intentos. Se desconocen las razones del fracaso, sin embargo, estas experiencias pudieron animar finalmente a ciudadanos de Bluefields, para la posterior fundación de las actuales universidades caribeñas BICU y URACCAN.

En la década de los 1980 se crearon la Región Autónoma Atlántico Norte (RAAN) y la Región Autónoma Atlántico Sur (RAAS), que cubren todo lo que antes era conocido como Costa Atlántica (actualmente denominada, Costa del Caribe). Es en estas dos regiones que se concentra la mayoría de las poblaciones indígenas y afrodescendientes de Nicaragua (PNUD, 2005). El Programa Educativo Bilingüe Intercultural (PEBI) se implementa en la Costa Caribe desde 1984, atendiendo en su lengua materna a niños y niñas miskitos, mayangnas y criollos en los niveles de preescolar y educación primaria, recibiendo educación en español y en su lengua materna, según la naturaleza de la comunidad atendida. El Programa de Educación Bilingüe tiene rasgos de ser intercultural, porque propicia la convivencia armónica entre las diversas culturas, en una situación de equilibrio, diálogo, respeto, tolerancia y encuentro entre las mismas, pero se debe evitar convertirla en un instrumento de "transculturización lingüística".

La interculturalidad es entendida como una relación entre diferentes culturas con la naturaleza y territorio; sin embargo, en Nicaragua este concepto, alcance y prácticas son poco asimilados y conocidos en la mayoría de las instituciones de educación superior.

La política de educación bilingüe como discurso ha tenido diferentes propuestas y acepciones a lo largo del siglo XX (Castillo Rodríguez y Ruiz López, 2016). García (2000:32) analiza cómo una estrategia de integración a través del bilingüismo, llamada método cultural, lejos de fortalecer la identidad cultural de los indígenas quechua, en Bolivia, tuvo resultados adversos. Un modelo educativo de tipo convencional va en detrimento de las minorías étnicas quienes pierden la confianza en sus valores y creencias, cayendo en indefensión de su identidad. Para Sandoval y Guerra (2007:5), el trasfondo de estas creaciones es la pérdida de la identidad indígena y su transformación hacia el mestizaje a través de la educación superior. "El sistema educativo, a la vez que coordina el saber opera como un filtro y una espiral de exclusión de otros saberes" Fornet (2004:22). 
Para Castillo Rodríguez y Ruiz López citando a Aguilar (2004,) "el discurso intercultural en educación tomó la forma de propuestas asimilacionistas, bilingües-biculturales, y más tarde bilingües-interculturales o interculturales bilingües" (93 pág.). La Declaración de las Naciones Unidas sobre los derechos de los pueblos indígenas, en su artículo 14 establece que: "los pueblos indígenas tienen derecho a todos los niveles y formas de educación del Estado. Todos los pueblos indígenas también tienen este derecho y también el de establecer y controlar sus sistemas e instituciones docentes, impartiendo educación en sus propios idiomas y en consonancia con sus métodos culturales de enseñanza y aprendizaje" (Art. 14). Así mismo, en el artículo 15 establece que "los pueblos indígenas tienen derecho a que la dignidad y diversidad de sus culturas, historias, tradiciones y aspiraciones queden debidamente reflejadas en todas las formas de educación y en la información pública" (URACCAN, 2016:8 p).

Con la promulgación de la Ley de Autonomía en 1987, la región del Caribe de Nicaragua adoptó un nuevo modelo de administración pública regional. El supuesto era facilitar por un lado el espacio público necesario para la representación efectiva de las distintas comunidades étnicas y, muy en especial, estaba supuesto a permitir la emergencia de un modelo de desarrollo económico y educativo que empoderara a estas comunidades para permitir una aprovechamiento más efectivo y racional del enorme caudal de riqueza y oportunidades para mejorar sus condiciones de vida.

Para dar inicio al cumplimiento del Artículo 8, inciso 2 de la Ley $\mathrm{n}^{\circ} 28$ (Estatuto de Autonomía de las Regiones de la Costa Atlántica de Nicaragua) se procedió a trasladar, los programas de educación bilingüe intercultural, bajo la administración directa de los gobiernos de las Regiones Autónomas. La Constitución de la República de Nicaragua, aprobada en 1987 y reformada posteriormente (última reforma en 2005), establece que "La educación tiene como objetivo la formación plena e integral del nicaragüense; dotarlo de una conciencia crítica, científica y humanista; desarrollar su personalidad y el sentido de su dignidad; y capacitarlo para asumir las tareas de interés común que demanda el progreso de la nación. Por consiguiente, la educación es factor fundamental para la transformación y el desarrollo del individuo y la sociedad" (Art. 116).

Se define la educación como "un proceso único, democrático, creativo y participativo que vincula la teoría con la práctica, el trabajo manual con el intelectual y promueve la investigación científica. Se fundamenta en los valores nacionales; en el conocimiento de la historia y de la realidad del país; de la cultura nacional y universal y en el desarrollo constante de la ciencia y de la técnica" (Art. 117).

La Ley General de Educación aprobada en marzo 2006 establece que: "la educación es creadora en el ser humano de valores sociales, ambientales, éticos, cívicos, humanísticos y culturales, está orientada al fortalecimiento de la identidad nacional. Reafirma el respeto a las diversidades religiosas, políticas, étnicas, culturales, psicológicas, de niños y niñas, jóvenes y adultos, que apunta al desarrollo de capacidades de autocrítica y crítica, de participación social desde el enfoque de una nueva ciudadanía formada en el respeto a la 
dignidad humana. La educación se regirá de acuerdo con un proceso de administración articulado, descentralizado, participativo, eficiente, transparente, como garantía de la función social de la educación sin menoscabo de la autonomía universitaria, el cual deberá interactuar con la educación no formal para alcanzar la formación integral.” (Art. 3).

En el Capítulo IV de la Ley General de Educación de 2006 (artículos 38 al 42), las Regiones Autónomas de la Costa del Caribe nicaragüense cuentan con un Sistema Educativo Autónomo Regional (SEAR), "orientado a la formación integral de las mujeres y hombres de los pueblos indígenas afro-descendientes y comunidades étnicas, basado en los principios de autonomía, interculturalidad, solidaridad, pertinencia, calidad, equidad, así como valores morales y cívicos de la cultura regional y nacional; comprometidos y comprometidas con el desarrollo sostenible, la equidad de género y los derechos de niños, niñas, adolescentes y jóvenes."

El Sistema de Educación Autonómico Regional (SEAR) es un subsistema educativo participativo, el cual se gestiona de manera descentralizada y autónoma y responde a las realidades, necesidades, anhelo y prioridades educativas de su población multiétnica, multilingüe y pluricultural. La misión y visión educativa nacional se orienta hacia la formación integral de niños y niñas, jóvenes y adultos, hombres y mujeres de la Costa del Caribe en todos los niveles del sistema educativo, así como hacia el respeto, rescate y fortalecimiento de sus diversas identidades étnicas, culturales y lingüísticas. El Estado proveerá de estos programas con los recursos apropiados para cumplir este fin (Art. 8).

Sobre la base de la Ley $\mathrm{N}^{\circ} 162$ de 1993, Ley de lenguas, en Nicaragua las comunidades de la Costa Atlántica tienen derecho a la preservación de sus lenguas. El Estado establecerá programas especiales para el ejercicio de este derecho, proporcionará los recursos necesarios para el buen funcionamiento de éstos, y dictará leyes destinadas a promover acciones que aseguren que ningún nicaragüense sea objeto de discriminación por razón de su lengua (Art. 2). Los Consejos Regionales Autónomos en coordinación con las autoridades educativas nacionales desarrollarán los programas educativos bilingües interculturales, respetando las normas básicas contenidas en la Ley, los que deberán responder a sus necesidades particulares y abarcarán su historia, geografía, recursos naturales, sus conocimientos y técnicas, sus sistemas de valores y sus aspiraciones sociales, económicas y culturales.

Dentro del marco jurídico internacional referido a tratados y convenios, el Programa de Educación Bilingüe se sustenta también en otros instrumentos:

1. Declaración Universal de Derechos Humanos. La Asamblea General de la Organización de las Naciones Unidas (ONU) proclamó el 10 de diciembre de 1948 la Declaración Universal de Derechos Humanos. 
2. Al respecto, el Artículo 26, numeral 4 establece: La educación tendrá por objeto el pleno desarrollo de la personalidad humana y el fortalecimiento del respeto a los derechos humanos y a las libertades fundamentales, favorecerá la comprensión, la tolerancia y la amistad entre todas las naciones y todos los grupos étnicos o religiosos; y promoverá el desarrollo de las actividades de las Naciones Unidas para el mantenimiento de la paz.

3. Convención relativa a la Lucha contra las discriminaciones en la Esfera de la Enseñanza del 21 de diciembre de 1965. Con su entrada en vigor el 4 de enero de 1969, el artículo $5^{\circ}$ establece que "los Estados parte se comprometen a garantizar a cada uno, sin distinción de raza, color u origen nacional o étnico, la igualdad de trato en el goce de los derechos civiles, políticos, económicos, sociales y culturales".

4. Declaración de Principios de la Cooperación Cultural Internacional. Aprobada en 1966 en la 14 Reunión de la Conferencia General de la UNESCO,

5. Convenio 169 de la Organización Internacional del Trabajo (OIT) sobre pueblos indígenas y tribales en países independientes, adoptado el 27 de junio de 1989 por la Conferencia General de la organización Internacional del Trabajo.

6. Declaración Universal de la UNESCO sobre la Diversidad Cultural del año 2001. En su Artículo 1 trata sobre la diversidad cultural, patrimonio común de la humanidad. La cultura adquiere formas diversas a través del tiempo y del espacio. Esta diversidad se manifiesta en la originalidad y la pluralidad de las identidades que caracterizan los grupos y las sociedades que componen la humanidad.

7. Al respecto, la Conferencia Regional de Educación Superior (CRES), del 2008, establece que "se deben promover la diversidad cultural y la interculturalidad en condiciones equitativas y mutuamente respetuosas [...]pertinentes con la diversidad cultural (URACCAN, 2016: 9)

8. Declaración de las Naciones Unidas sobre los Derechos de los Pueblos Indígenas. Resolución aprobada por la Asamblea General de la ONU el 13 de septiembre de 2007.

\section{La multiculturalidad e interculturalidad en la educación}

En muchas ocasiones, los términos multiculturalidad e interculturalidad se utilizan indistintamente, sin embargo, para muchos autores ambos tienen diversos significados y alcances que son aplicados según la conveniencia o contexto. Interculturalidad, es más integradora y debe ser entendida como un proyecto político, social epistémico y ético (Tubino 2005:24-28). 
Las expresiones educación multicultural y educación intercultural, se emplean con otras

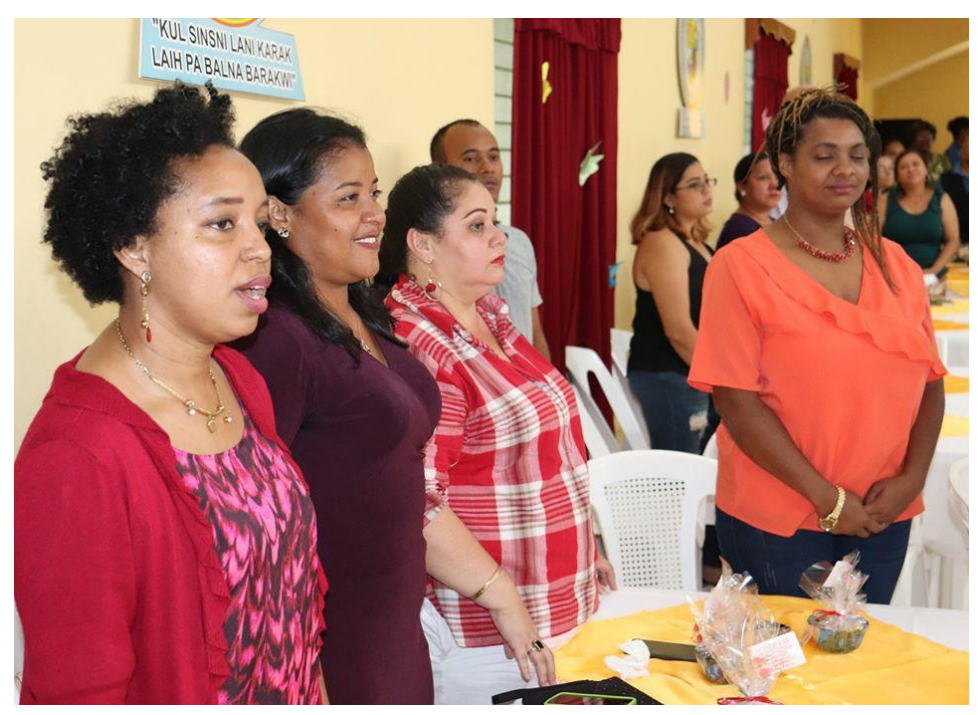
expresiones próximas como educación multiétnica, educación multirracial, educación multilingüe $\mathrm{y}$ educación pluralista. Cualquiera de estos términos o acepciones implica reconocimiento, enriquecimiento mutuo y valores culturales (Méndez, 2012:24). Otros conceptos son "relación y aceptación entre culturas", "afirmación identitaria a partir del conocimiento de una cultura hermana", "respeto entre culturas".

Fig. 1. Miembros del personal docente de BICU. (Archivo BICU).

En cuanto a Multiculturalidad, para Walsh 2009, citado por Tubino (2012), multiculturalidad es descriptivo y "[...] se refiere a la multiplicidad de culturas existentes dentro de un determinado espacio [...] sin que necesariamente tengan una relación entre ellas". "La interculturalidad [...] se refiere a complejas relaciones, negociaciones e intercambios culturales y busca desarrollar una interacción entre personas, conocimientos, prácticas, lógicas, racionalidades y principios de vida culturalmente diferentes (pag.19).

En los Estados Unidos, desde el año 1979, “el Consejo Nacional para la Acreditación de la Formación de Profesores en 1977 puso en vigencia la norma de educación multicultural, lo cual vino a incentivar la educación multicultural en todos los contenidos de estudios profesionales" (Banks, 1993:3). La educación multicultural tuvo su nacimiento en Estados Unidos en la década de 1950, mientras que en Europa irrumpió veinte años después, con la similitud que surgen para responder a demandas educativas y evitar la supremacía y conflictos de culturas. Para Banks (1993:2), la educación multicultural no es asunto étnico ni de género sino "un movimiento diseñado para empoderar de conocimientos a todos los estudiantes, para que sean ciudadanos activos en un mundo cada vez más profundamente problemático y polarizado".

Mientras tanto Méndez (2012:21) plantea que por educación multicultural debe entenderse: No solo el espacio donde convergen estudiantes con diferentes culturas, etnias y saberes, sino que se les respetan y promueven. Para Aguado (1990) "es un espacio para promover la igualdad de acceso a educación universitaria de calidad, la defensa de los valores democráticos fundamentada en el respeto del otro" (p. 2). 
En algunos ambientes multiculturales en los Estados Unidos es frecuente que muchos docentes alegan no disponer de programas ni planes para educación multicultural porque la presencia de estudiantes blancos prevalece sobre las otras minorías étnicas (Banks,1993:2). Universidades en Estados Unidos como Berkeley en California, Minnesota, y de Stanford han tenido avances significativos en cuanto a educación multicultural, propiciando cambios en sus objetivos y contenidos curriculares al incluir contenidos curriculares y estudios étnicos obligatorios como requisitos en sus carreras y cursos (p. 3).

Con relación a la Educación multicultural, Gómez, et al. (2008: 10) señala: "Se debe aspirar a una educación que desarrolle valores como la tolerancia, el respeto y la sensibilidad ante los distintos modos de vida y de cultura; formar juicios independientes como parte de la capacidad autocrítica promovida bajo el modelo multicultural".

Al margen de preceptos tan variados, lo importante es diferenciar la multiculturalidad, referida al dominio y control de un grupo numérica y culturalmente poderoso, con respecto al de la convergencia de culturas y prácticas de respeto mutuo en un espacio determinado, por ejemplo, un territorio, comunidad, o universidad. En el caso de BICU, sus programas y currículos ¿están orientados a la interculturalidad y cosmovisión de los pueblos indígenas y afrodescendientes?

En esta época de globalización y cambios mundiales de modernización, donde prevalece la idea de alcanzar el bienestar y desarrollo basados en la óptica occidental, por excelencia se siguen patrones socioeconómicos y culturales propios de la cosmovisión de países con mayor desarrollo e influencia (Gómez, et al., 2008:2). Con la globalización, esta modernidad entra en crisis: estamos viviendo su crisis, un proceso de cambios del que no sabemos qué de nuevo va a surgir. Una consecuencia de esta crisis es la incertidumbre, que nos ha de llevar a comprender la fragmentación del mundo y nuestras limitaciones en interpretarlo" Cabrero (2008:4).

Uno de los riesgos de la "modernidad" es que se trate de imponer los criterios de las mayorías sobre quienes son y piensan diferentes, como las minorías étnicas, y hacer educación igual a la que hacen otras universidades. De esta forma homogenizan programas y currículos educativos o al menos tratan de aproximarse a lo que hacen las del primer mundo, cuya realidad y pertinencia difiere al de nuestras comunidades. Es por ello que, en relación a criterios de mayorías, Gómez-Buendía et al (2008:4), dejan claro que dicho principio no implica el ejercicio absoluto del poder sobre las minorías, aun cuando pasa por las violaciones de los derechos humanos fundamentales de estas minorías. 


\section{Interculturalidad}

La interculturalidad es un término discursivo que en las últimas décadas ha sido utilizada muchas veces por conveniencia y según el contexto donde se aborde. Uno de los contextos más utilizados es el educativo. Ante la posibilidad de manipulaciones del término "interculturalidad", Tubino (2005:24-28), acertadamente plantea y diferencia la interculturalidad "funcional, porque "no cuestiona las reglas del juego y es perfectamente compatible con la lógica del modelo neoliberal existente", de la interculturalidad entendida como proyecto político, social, epistémico y ético.

En la universidad URACCAN (2016) conciben la interculturalidad como un proceso permanente de construcción, establecimiento y fortalecimiento de espacios de diálogo, comunicación e interacción horizontal de doble vía, entre personas, comunidades y pueblos de diferentes culturas. La interculturalidad comprende el desarrollo de procesos de toma de decisiones conjuntas en igualdad de condiciones. Su finalidad es la de la promoción y práctica de la equidad, el respeto, la comprensión, la aceptación mutua y la creación de sinergias para el establecimiento de sociedades inclusivas, no discriminatorias y libres de racismo $(41 \mathrm{p})$.

Para Alsina (2001:8), la interculturalidad implica un cambio de mirada del mundo en dos aspectos: la mirada endógena y la mirada exógena". Por su parte, para Walch la "interculturalidad es, cada vez más, el término usado para referir a estos discursos, políticas y estrategias de corte multicultural-neoliberal (2009:8). En países como México donde la interculturalidad desde el ámbito educativo se debate desde hace más de dos décadas, el reto sigue siendo cómo darle una aplicación práctica efectiva a la interculturalidad vista más desde los componentes pedagógicos, que desde las políticas del bilingüismo (Castillo Rodríguez y Ruiz López, 2016). En Nicaragua el principio de interculturalidad establecida en la Ley $\mathrm{N}^{\mathrm{o}}$. 704, ley creadora del sistema para el aseguramiento de la calidad de la educación y reguladora del Consejo Nacional de Evaluación y Acreditación (CNEA), se conceptualiza como "La diversidad étnica, lingüística, cultural, técnica, científica y humanista y reconoce las diferentes formas educativas y enfoques que se implementan en el territorio nacional".

\section{Evaluación de modelos educativos}

Conceptualmente, la evaluación es un término que tiene muchas acepciones las cuales muchas veces se interpreta como momentos de "inquisición" por parte de los sujetos que son evaluados y no como un componente importante dentro del proceso para la mejora de los modelos educativos. Moreno, Espinosa, Solano y Fresán (2016) citando a varios autores aportan definiciones importantes al respecto: Ralph Tyler (1986) concibe la evaluación como el proceso de determinar hasta qué punto se están alcanzando realmente los objetivos educativos. Otra definición muy aceptada, que ha sido sugerida por investigadores prestigiados, como Cronbach (1963), Stufflebeam (1969) y Alkin (1969), se refieren a la evaluación como el mecanismo que permite proporcionar información para la toma de decisiones $(31 \mathrm{pg})$. 
Un proceso de evaluación debe servir para replantear el rol de la universidad, su pertinencia social en la búsqueda de las mejoras continuas para asegurar una educación integral y de calidad. Cabe señalar que no basta hacer una evaluación meramente por cumplir una actividad establecida como parte de un proceso de planificación, sino para hacer las mejoras pertinentes a lo evaluado. Ante las diferentes implicancias que puede derivarse de una

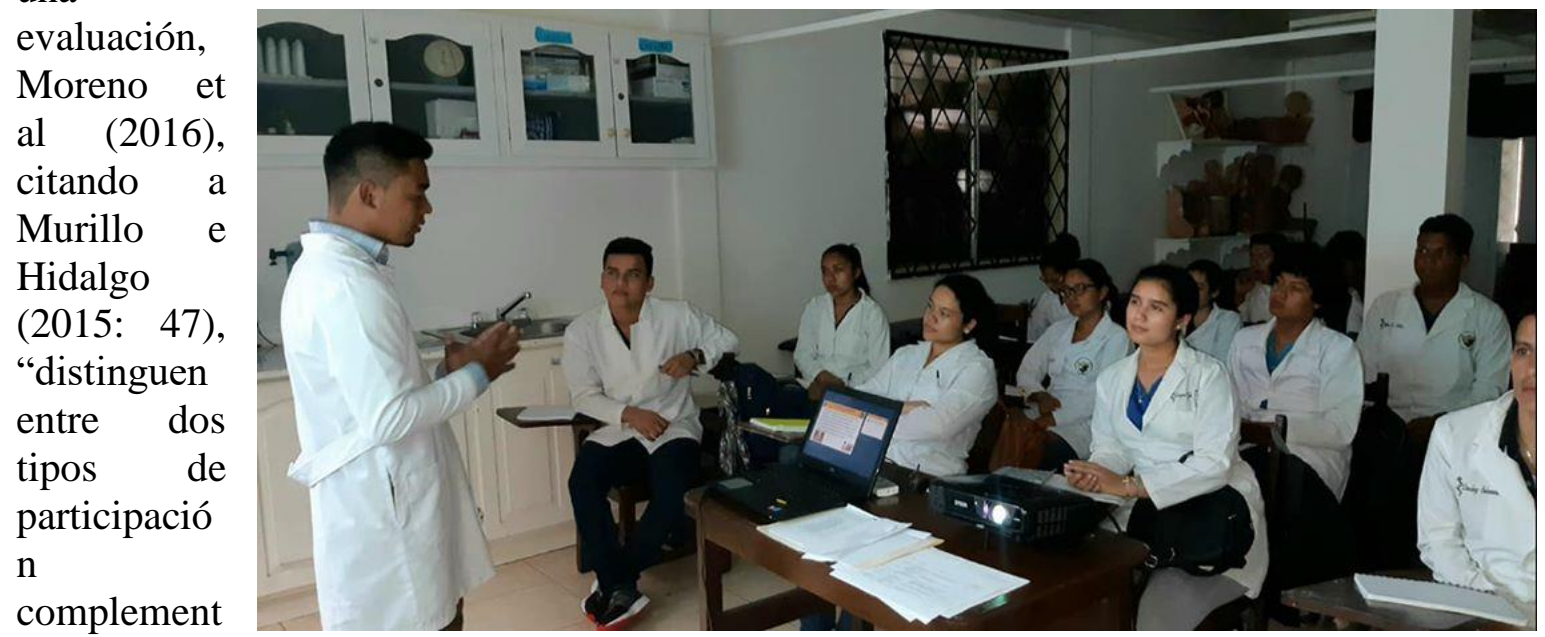

arios:

Fig. 2. Estudiantes de medicina costeños. (Archivo BICU).

a) Evaluación participativa práctica: implica a sus estudiantes en la toma de decisiones y la resolución de problemas. Se trata de un enfoque que incluye los principios democráticos en su práctica evaluativa, pero no tiene un compromiso explícito con el cambio social.

b) Evaluación participativa transformadora: tiene como objetivo el logro del cambio social, empoderando a los participantes de la evaluación, y pone especial atención en la inclusión de aquellos participantes tradicionalmente oprimidos (pp. 32-33).

\section{Modelos educativos universitarios y sus características}

Aunque en la actualidad existen diversas acepciones referidas a lo que se entiende por modelo educativo, la mayoría coincide en que un modelo educativo es la base para guiar el aprendizaje del estudiante, especialmente ante nuevos paradigmas educativos y pedagógicos. Para Tünnerman Bernheim (2008) "El modelo educativo es la concreción, en términos pedagógicos, de los paradigmas educativos que una institución profesa y que sirve de referencia para todas las funciones que cumple (docencia, investigación, extensión, vinculación y servicios), a fin de hacer realidad su proyecto educativo". El mismo autor en cuanto a definición de modelo educativo en el Instituto Politécnico Nacional de México, sostiene que es "una representación de la realidad institucional que sirve de referencia y también de ideal. Como tal, va enriqueciéndose en el tiempo y sustenta el quehacer del instituto" (p. 15). 
Un modelo educativo es la expresión formal y filosófica de una universidad que, a través de su misión y misión institucional, guía los procesos de formación técnica y profesional de sus protagonistas y la distingue de otras universidades, constituyéndose en sí misma como un referente de calidad (Núñez, 2010). Tünnerman Bernheim por su parte destaca que un modelo educativo, "deberá proyectarse en todas las funciones básicas como un modelo de la universidad: docencia, investigación, extensión, vinculación y servicios" (p. 18). En México, el modelo educativo vigente al 2018 para todos los niveles formativos, está estructurado en ejes entre las cuales incluye planteamiento curricular, la Escuela al centro del sistema educativo, formación y desarrollo profesional del maestro, inclusión y equidad y gobernanza del sistema educativo (SEP,2017). De estos, el eje de inclusión y equidad es muy importante por cuanto además de ver con derechos para grupos marginados, debería ser un principio infaltable de toda instancias de educación intercultural.

\section{El modelo educativo de universidades interculturales de México}

Los modelos educativos en universidades interculturales mexicanas (Rojas Cortez, 2016) organizan sus componentes mediante ejes trasversales y lineales entre las que se destacan "Relación estrecha con las comunidades, mediante prácticas sociales, prácticas comunitarias, estancias comunitarias o como opción de trabajo terminal, alcanzando propósitos tales como, el diálogo de saberes, el arraigo de los alumnos a sus comunidades o impulsar un desarrollo comunitario" (p. 191).

En el Estado de Chiapas, México, existen experiencias positivas del Consejo Nacional de Fomento Educativo (CONAFE) relacionadas al modelo de educación comunitaria (Carratalá, 2012). El modelo consiste en la selección y entrenamiento a jóvenes de las comunidades rurales con habilidades y competencias interculturales, para ser instructores comunitarios en la Modalidad de Atención Educativa a Población Indígena (MAEPI) que les ayude a la promoción y fortalecimiento de la diversidad cultural en el contexto y ambiente educativo multicultural y complejo (Gómez, 2009:4). La misma autora citando a Casillas y Santini, (2006) señala que el modelo de las Universidades Interculturales dispone de eje sustantivo junto con la lengua y la cultura, mediante la Proyección Social o por ellos llamada vinculación Comunitaria.

\section{El Modelo Educativo de la Universidad Nacional Autónoma de Nicaragua (UNAN- Managua).}

El modelo educativo de la UNAN Managua, hasta antes de su última reforma del 2013, era de "corte napoleónico; currículos con objetivos orientados al conocer y saber hacer" (UNAN-MGA, 2018). Actualmente el nuevo modelo educativo presenta cambios en cuanto a la didáctica, caracterizada por un proceso de enseñanza aprendizaje dinámico. En cuanto a los estudiantes, el modelo es interactivo para con sus saberes, auto-reflexivo con lo que aprende, y aplicativo en los conocimientos para su futuro desempeño profesional, mediante la; evaluación de la competencia. 
Los componentes esenciales del nuevo modelo educativo centrado en el estudiante son: modelo pedagógico, modelo curricular y modelo didáctico. El modelo pedagógico tiene como pilares fundamentales la conceptualización y visión del proceso educativo que concibe la enseñanza como un proceso dinámico por cuanto combina la experiencia y conocimientos previos del estudiante con cambios en el rol del docente y los contenidos. Este dinamismo permite un cambio de enfoque del proceso de enseñanza aprendizaje autónomo en doble vía lo cual facilita al estudiante aprender a Hacer, Ser y Emprender, para enfrentar nuevos retos en la vida en cuanto a la creación y convivencia para la trasformación social. En este sentido, el modelo también implementa el uso de la Tecnologías de Información y Comunicación (TIC), como herramienta clave para desarrollar nuevos conocimientos de manera integral en cuanto al desarrollo de capacidades en la formación profesional.

En cuanto al modelo curricular es global e integrado, flexible como marco de orientación y reflexión para mejorar la práctica a través de la reflexión. El docente que es el investigador continuamente trasforma los ambientes del aprendizaje y la formación permanente e integral para la transformación social. Por otra parte, el modelo didáctico permite que el docente sea un motivador y admita la elaboración y puesta en práctica de guías de cuestionamiento de la relación entre el ser humano y su entorno. De esta manera se facilita el aprendizaje colaborativo, el estudio de casos proyectos, trabajo de campo y conferencias. Para la UNAN-Managua, en la implementación de este modelo educativo, la investigación es clave para que el estudiante pueda desarrollar un aprendizaje autónomo y tener criterios para diferenciar, seleccionar y apropiarse de nuevos conocimientos, valorando la confiabilidad de las fuentes de información.

Por lo antes expuesto, a partir del año 2015 se desarrolla el Modelo de Gestión de la Investigación, Modelo I+D+i de la UNAN-Managua, el cuál según Pedroza Manuel (2015): Representa la visión prospectiva de los procesos de investigación científica de la UNANManagua, articulados a los procesos de innovación tecnológica, socio-productiva, organizacional y educativa. El Modelo está centrado en la Misión, Visión y Valores de la UNAN-Managua (pág. 3). En cuanto al proceso de aprendizaje del modelo didáctico, éste se centra en el estudiante y en el desarrollo de procedimientos a fin de que pueda desarrollar, habilidades, estrategias y técnicas que le permitan ser y aprender a conocer, hacer, emprender y crear. De esta manera se pretende que los egresados sean capaces de enfrentar la vida y desarrollar procesos continuos de actualización y adquisición de conocimientos. Se garantiza así, que los egresados adquieran herramientas conceptuales, procedimentales y actitudinales, para la resolución de problemas ante la demanda de un mundo cambiante.

Al analizar el modelo educativo de la UNAN-MANAGUA si bien está centrado en el estudiante, esta conceptualizado para atender de manera homogénea a todos sin excepción alguna. El currículo no incluye indicadores ni criterios interculturales referidos a pueblos indígenas ni afrodescendientes. 


\section{Modelo Educativo de la Universidad de las Regiones Autónomas de la Costa Caribe Nicaragüense (URACCAN).}

Atendiendo el Plan Educativo Institucional (URACCAN, 2016:37-39), el modelo tiene tres componentes caracterizados textualmente así: con relación al modelo curricular es entendido como el proceso de construcción educativo y cultural, en el cual se organiza un conjunto de experiencias y prácticas. El currículo está organizado de manera innovadora, flexible, interdisciplinaria e integradora de conocimientos y experiencias de aprendizaje, bajo el criterio de interculturalidad en el cual se incorporan las historias, idiomas, saberes. El currículo intercultural recupera las historias, idiomas, saberes, conocimientos, prácticas y tecnologías de los pueblos. Finalmente, el modelo didáctico está centrado en quien estudia y su aprendizaje, el mismo responde al enfoque del socio-constructivismo, teniendo en cuenta que la cultura de origen forma parte fundamental de los conocimientos y actitudes previas. Los saberes y conocimientos históricamente acumulados por cada pueblo originario son los pilares inmediatos de donde se parte. Recupera todos estos valores, sin excluir los nacionales $\quad \mathrm{y}$ universales, dentro de un contexto comunitario intercultural, revitalizando esta forma los saberes comunitarios y las prácticas exitosas, de manera interdisciplinaria.



Fig. 3. Profesora impartiendo clase en una universidad costeña. (Archivo BICU).

\section{MODELO PEDAGÓGICO}

Promueve la interacción (docente-estudiante y estudiante-estudiante), lo cual es una estrategia pedagógica fundamental. De igual manera facilita un ambiente de aprendizaje adecuado y cómodo, donde cada persona se siente importante, protagonista, respetada y valorada por igual y puede expresar libremente su opinión y ser escuchado, aceptado y criticado de manera constructiva. La planificación didáctica es abierta, flexible y diversificada. Problematiza los contenidos y los explica desde diversas miradas culturales. Integra la participación de la comunidad como fuente de conocimiento y de conexión con la realidad del contexto y atendiendo la diversidad desde la igualdad de derechos. 
El modelo desarrolla estrategias para fortalecer el bilingüismo oral y escrito, tanto en la lengua nacional como la originaria, y la comunicación. El plan de estudio es diseñado para contribuir al desarrollo de la profesión y de la comunidad.

\section{El surgimiento de las universidades (¿comunitarias e interculturales?)}

Los programas educativos de las universidades convencionales limitan de alguna manera los valores culturales e identidad al promover valores exógenos del "primer mundo", minimizando los conocimientos y saberes de los estudiantes pertenecientes a minorías étnicas. Los currículos académicos de alguna manera son homogéneos (Näslund-Hadley, Meza, Arcia, Rápalo, \& Rondón, 2012). Los currículos de las carreras están diseñados de manera que los estudiantes pertenecientes a minorías étnicas deben someterse a reglas y normativas homogéneas establecidas por cada casa de estudios. Esto genera desiguales condiciones para los estudiantes indígenas y afrodescendientes, que se acentúan aún más ante la falta de comunicación y expresión en su lengua materna (PNUD, 2005).

Dado que generalmente la lógica educativa de las instituciones públicas de educación superior convencionales no está pensada en el desarrollo inclusivo ni equitativo, se hace necesario la búsqueda de otras opciones educativas para subsanar estas brechas. Al respecto de convencionalidades en la educación superior, Casillas y Santini (2009) plantean que "las Universidades Públicas la formación para todos los actores sociales y su integración al desarrollo del país, en estas generalidades quedan, patrimonial y culturalmente marginados, las comunidades rurales e indígenas" (p. 14). En nuestro juicio, al creerse que la formación en las universidades públicas debe ser de carácter general, los abordajes educativos son trasversales y culturalmente etnocentristas.

El carácter de universidades comunitarias en Latinoamérica es incipiente, excepto en Brasil y México donde tienen casi dos décadas de existencia. Desde el punto de vista de interculturalidad, sea de hecho o como proyecto, México (Rojas, Cortez, 2016:184) reporta la existencia de doce universidades interculturales que se aglutinan bajo la Coordinación General de Educación Intercultural y Bilingüe de la Secretaría de Educación Pública (SEP, 2017).

Es importante señalar que en Nicaragua no existe una red de universidades comunitarias e interculturales. Tanto BICU como URACCAN están aglutinadas en el Consejo Nacional de Universidades (CNU). Sin embargo, creemos que ambas deberían crear su propia red para visibilizarse, nacional e internacionalmente, ante universidades cuya filosofía institucional sea similar. En cuanto a las características relativas a diversidad en las universidades interculturales, en Casillas y Santini (2009) hay que destacar tres aspectos:

1) Introducen diversidad en términos de oferta educativa;

2) Permiten el establecimiento de plataformas para la investigación y el desarrollo de saberes diversos y

3) Establecen un modelo de organización académica diferente, con grandes potencialidades tanto en términos de formación, como de artificio en desarrollo. 
A nuestro criterio, una universidad intercultural es aquella cuyo "proyecto educativo rescata, promueve, fortalece, visibiliza e integra la diversidad y el diálogo de los saberes ancestrales, culturales, lingüísticos y la cosmovisión de las comunidades de su entorno". Aun con los rasgos antes mencionados para las universidades interculturales, Rojas Cortez (2016) advierte sobre la existencia de "iniciativas de educación superior en México de bases comunitarias no aglutinadas al SEP (2017), cuyos modelos educativos contienen propuestas de corte interculturales pero que al final son proyectos descolonizadores y emancipatorios" (p. 185). En el caso de las universidades interculturales de México, Casillas y Santini (2009), el surgimiento de universidades interculturales tiene características propias y distintivas; destacándose las siguientes:

- Están próximas en ubicación y en cultura a las comunidades indígenas.

- Son fuente importante de generación y diseminación de conocimientos pertinentes para las mismas comunidades, y espacios de formación de nivel superior para estudiantes provenientes del entorno próximo.

- Están diseñadas para convertirse en instrumentos de desarrollo en manos de los propios hijos de las comunidades.

- Por último, sustentan sus propuestas en un diálogo permanente con las propias comunidades y sus saberes.

En Nicaragua, la carencia de estrategias educativas para la atención y superación de las brechas socioculturales y educativas entre poblaciones mayoritarias versus minorías étnicas multiculturales y multilingües, hace que la calidad de la educación superior de estas minorías étnicas, a nivel nacional quede invisibilizada. La falta de interacción con estos grupos marginados se constituye en la misión y principal justificación de la creación y existencia de las universidades interculturales (Casillas y Santini, 2009:14). La continuidad de una lógica educativa universitaria de mayorías étnicas y lingüísticas como un todo, ha supuesto un riesgo y amenaza para la profesionalización de estudiantes de las minorías étnicas y un impacto negativo en la función social de la universidad. Aun cuando en sus inicios BICU de manera tácita no abordaba algunos rasgos de interculturalidad, en sus reformas estatutarias del 2011 retoma e introduce en el artículo 3 de sus estatutos, valores y compromisos encaminados a poner en práctica compromisos interculturales, destacando entre estos:

a) La promoción de una conciencia de respeto a la dignidad humana a favor de la convivencia nacional del individuo y de los pueblos.

b) La promoción de una conciencia crítica y creativa en el proceso de transformación social.

c) La promoción de la investigación, documentación, publicación y enseñanza de la historia, cultura, tradiciones, idiomas o lenguas de los pueblos indígenas, afrodescendientes y mestizos de la Costa Caribe Nicaragüense en particular y del pueblo nicaragüense en general. 
d) El fortalecimiento del proceso del Régimen Autonómico y el desarrollo de la Costa Caribe Nicaragüense.

En México, Casillas y Santini, reportan que la justificación para la creación de universidades interculturales obedece a que los jóvenes que logran salir de sus comunidades acceden a estudios superiores en las universidades existentes y concluyen su carrera exitosamente, pero cuentan con una formación generalmente ajena a las necesidades de desarrollo de la región que habitan. Esta formación les impone una visión ajena a sus valores y prácticas culturales, y terminan por buscar horizontes de trabajo y de vida alejados de su entorno. Esto provoca que dichos profesionales abandonen y rechacen totalmente la práctica de su lengua, así como sus nexos con la cultura de su medio y no puedan concretar un compromiso con el desarrollo de su región" (p. 142). En consecuencia, de la existencia de universidades que atienden a grupos poblacionales marginados, según el Instituto Internacional de la UNESCO para la Educación Superior en América Latina y el Caribe, "existen más de cincuenta instituciones de educación superior dedicadas a las comunidades indígenas y afrodescendientes" (BICU, 2017: 9).

$\mathrm{Al}$ observar en el párrafo anterior lo expuesto por la UNESCO, queda en evidencia que esa "existencia" no destaca el tipo de IES desde el punto de vista comunitario ni el intercultural, sino que refiere únicamente a la prestación de servicios para grupos marginados. Este auge abre oportunidades de acceso para poblaciones indígenas y afrodescendientes históricamente marginadas, aunque con ciertas dudas respecto a la pertinencia de sus programas en beneficio de estas poblaciones. Mientras en Brasil se contabilizan 17 universidades comunitarias públicas de derecho privado no confesionales (Netto, 2008), en Nicaragua solamente las universidades caribeñas, BICU y URACCAN, se declaran como comunitarias e interculturales. Al respecto, Casillas (2012), citando a Cunningham (2002), la Universidad de las Regiones Autónomas de la Costa Caribe Nicaragüense (URACCAN), se define como "una universidad comunitaria intercultural de los pueblos indígenas y comunidades étnicas" (pág. 91). En términos similares, BICU en el Arto 2 de sus Estatutos vigentes se define como "Universidad Autónoma Comunitaria, intercultural patrimonio de los Pueblos Indígenas, Afrodescendientes, Mestizos de las Regiones Autónomas de la Costa Caribe".

Quizá por lo reciente de la existencia de universidades comunitarias por un lado y de las interculturales por otra parte, y otras que se declaran sea como comunitarias o como interculturales, y sin entrar en debate de si lo comunitario implica lo intercultural o viceversa, en nuestra opinión estos términos muchas veces podrían manejarse, discrecional e indistintamente, a conveniencia de partes. Por las características socioculturales propias de la Costa Caribe surge la necesidad de construir un concepto integrado de "comunitaria e intercultural". La existencia e importancia de esta conceptualización radica en la necesidad de establecer una diferenciación de universidades comunitarias e interculturales respecto a otras convencionales, en virtud del perfil de sus modelos educativos y pertinencia etnosocial de las primeras. 
Para la Universidad de las Regiones Autónomas de la Costa Caribe de Nicaragua (URACCAN), por Universidad Comunitaria Intercultural, se puede conceptualizar como aquellas "que se inscribe en el marco del reconocimiento y ejercicio de derechos humanos colectivos, que los pueblos indígenas y comunidades étnicas han reivindicado (URACCAN, 2016:21).

\section{Rasgos y características de Universidades Comunitarias.}

Aun cuando el concepto y definición de universidad comunitaria está en debate permanente y quizá para muchos, esto está íntimamente ligado a la interculturalidad como un todo o al menos un eslabón de ella, por ello es necesario intentar la búsqueda de ciertos rasgos para

la conceptualización conjunta de dichos rasgos, pero sin menoscabo de sus propuestas educativas.

Según Netto (2008), una de las peculiaridades que distingue a las universidades comunitarias en Brasil, y que merecen por tanto una distinción conceptual, "es el reconocimiento de la función social e histórica que ese conjunto de instituciones desempeñó en la interiorización de la educación superior" (pg. 37). Al parecer, el término "interiorización de la educación" se refiere a que la universidad sale de la parte urbana, de las principales ciudades y llega lugares alejados. En otras palabras, a nuestro criterio, la municipalización de la educación es un criterio importante para caracterizar a una universidad de "comunitaria".

Para Gómez (2009), "las propuestas educativas basadas en la educación comunitaria son la mejor vía para la formación de un sujeto autónomo". El autor destaca que los programas del Consejo Nacional de Fomento Educativo (CONAFE) en México, "aprovechan la diversidad cultural como una ventaja pedagógica, y sustenta su metodología en el desarrollo de competencias básicas para la vida" (pág. 4). Aunque Casillas y Santini (2009) no definen lo que es una universidad comunitaria, los rasgos que establecen para una universidad intercultural coinciden con algunos de los rasgos de universidad comunitaria planteados por Netto. Para estas autoras, los rasgos de universidad intercultural incluyen:

- Están próximas en ubicación y en cultura a las comunidades indígenas.

- Son fuente importante de generación y diseminación de conocimientos pertinentes para las mismas comunidades, y espacios de formación de nivel superior para estudiantes provenientes del entorno próximo.

- Están diseñadas para convertirse en instrumentos de desarrollo en manos de los propios hijos de las comunidades.

- Sustentan sus propuestas en un diálogo permanente con las propias comunidades y sus saberes.

- Como un ejemplo de instrumento de desarrollo, en la Universidad Indígena Intercultural de Kawsay, Bolivia, en sus programas y currículos trabajan temáticas de Pedagogía Intercultural, Derechos Humanos, Derechos Indígenas, Ecoturismo Comunitario, Gobiernos Comunitarios y Eco producción Comunitaria. 
Para Casillas et al (2016), Lo comunitario busca construir los procesos de aprendizaje a través de una construcción conjunta entre participantes, comunidad, tutores, facilitadores de curso y equipo. (pg. 76).

Oficialmente, en la BICU el término Comunitario e Intercultural fue incluido en la primera reforma de los estatutos, publicada en la Gaceta diario oficial $\mathrm{N}^{\circ} 187$ del 5 de octubre del 2011, que en el artículo 2 establece: "Bluefields Indian \& Caribbean University es una Universidad Autónoma, Comunitaria y de derecho público, patrimonio de los Pueblos Indígenas, Afro - Descendientes y Mestizos de las Regiones Autónomas de la Costa Caribe Nicaragüense, apartidaría, multiétnico, intercultural”.

Con relación al derecho público de las Universidades Comunitarias en Brasil, para Netto (2008) hay ambigüedad al decir que "son públicas de derecho privado, gozan de filantropía, su gestión es colegiada, los alumnos pagan aranceles, sin embargo; el patrimonio adquirido es público" (p. 39). A diferencia del caso del Brasil, la BICU es comunitaria de derecho público, no privado; es decir, sin fines de lucro, establecidos en los Estatutos. Adicionalmente, si bien en la BICU muchos estudiantes pagan un arancel por estudios, prácticamente hay gratuidad de éstos al ser subsidiadas todas las carreras con el $6 \%$ constitucional que recibe del erario nacional. Así se evitan de pagar unos U\$ 45 dólares mensuales que es el promedio de pago de arancel en una universidad privada en Nicaragua.

Insistiendo en los rasgos que determinan, en Brasil, la naturaleza de una universidad con perfil comunitario, éstos se caracterizan porque han surgido de la asociación de liderazgos regionales y en municipios alejados donde no llegan otras universidades públicas ni privadas. Las particularidades de estas universidades son:

a. surgen gracias al esfuerzo conjunto de diversos actores de la sociedad;

b. apoyan el desarrollo socioeconómico de las comunidades y

c. geográficamente están ubicadas en lugares poco atendidas por los gobiernos.

Según nuestro criterio, los rasgos antes planteados coinciden plenamente con el surgimiento de BICU, agregándose en nuestro caso los aportes organizativos, colaborativos y el liderazgo asumido por actores civiles claves, así como los Consejos Municipales, en la fundación de todas sus sedes; y por estos criterios es que se refuerza su derecho al patrimonio de la universidad comunitaria. Cabe destacar que BICU, en el artículo 3 de sus Estatutos reformados en el 2016, de manera tácita y clara establece sus compromisos en cuanto al apoyo y las transformaciones a favor de los pueblos indígenas, afrodescendientes y mestizos de la Costa Caribe Nicaragüense en particular, y del pueblo nicaragüense en general.

Otros rasgos coincidentes del carácter comunitario es que en la creación de cada una de las ocho sedes de la BICU hubo aportes claves de los líderes y representantes de instituciones civiles, eclesiásticas, municipales y gubernamentales, para que, a través de las sedes 
distribuidas en la Costa Caribe Sur y Caribe Norte, puedan atender a estudiantes provenientes de zonas geográficas adonde no llegan las universidades públicas ni privadas. En el ámbito organizativo y colaborativo de las comunidades donde se fundaron las sedes de BICU, los terrenos fueron donados por la comunidad o, en su defecto, por los Consejos Municipales; se conformaron asociaciones pro-BICU en cada municipio. Es por ello que $\mathrm{BICU}$ responde al desarrollo comunal, territorial y regional, estableciendo en sus estatutos que esta universidad es patrimonio de los pueblos y comunidades indígenas, afrodescendientes y mestizas.

En la BICU hay participación y representación de los pueblos y comunidades en el Consejo Universitario, a través de una Junta Directiva conformada con un representante de cada una de las seis etnias, lo que les da un carácter de pertenencia.

Con todos las características y rasgos y criterios antes mencionados, a juicio nuestro, la base para conceptualizar una universidad como comunitaria es el carácter del derecho del cual emana un fin y el tipo de propiedad. En tal sentido, una Universidad Comunitaria en esencia es aquella de Derecho público sin fines de lucro, de patrimonio Comunitario, organizada y constituida participativamente con diferentes actores cuyos fines responden prioritariamente, tanto a escala temporal como geográfica, al desarrollo socioeconómico con identidad de las Comunidades de su entorno"

\section{CONCLUSIONES}

La multiculturalidad y el multilingüismo de los pueblos y comunidades en las Regiones Autónomas del Caribe nicaragüense constituyen un reto socioeconómico y cultural importante para el Estado nicaragüense, por cuanto no solamente debe restituirles derechos históricamente marginados, sino que deben tener congruencia con la identidad cultural y cosmovisión de los pueblos y comunidades indígenas, afrodescendientes y mestizos que en ella habitan. Un ejemplo de ello es el Programa de Educación Bilingüe que, aunque tiene rasgos de interculturalidad, se debe cuidar que éste se convierta en un instrumento de transculturización lingüística.

Los términos multiculturalidad e interculturalidad se utilizan indistintamente. Sin embargo, creemos que los diversos significados y alcances, en muchas ocasiones son aplicados a conveniencia y/o según el contexto. Mientras la multiculturalidad atañe a la convergencia geográfica de diferentes etnias y culturas, la interculturalidad es más integradora y debe ser entendida como un proyecto político, social epistémico y ético (Tubino 2005:24-28).

Un modelo educativo, por cuanto guía los procesos de formación técnico profesional de sus estudiantes, requiere de un proceso constante y sistemático de evaluación participativa y efectiva. Estas acciones son útiles para la realización de transformaciones y replanteamientos que respondan a la educación con pertinencia en una nueva época.

Aun cuando el propósito de la presente investigación no es indagar a profundidad cada componente de los respectivos modelos educativos y académicos en cada caso, se evidencia 
que, en las universidades interculturales, el modelo se basa en el ocio constructivismo y que de alguna manera recogen algunos criterios de interculturalidad referidos a grupos vulnerables y marginados. En el caso de la Universidad Pública UNAN-MANAGUA, el modelo es constructivista. El currículo es homogéneo para todos los estudiantes sin excepción y no incluye indicadores ni criterios interculturales referidos a pueblos indígenas ni afrodescendientes en particular.

A nivel mundial han surgido universidades comunitarias e interculturales, comunitarias, y otras, únicamente interculturales, todas de origen reciente que merecen ser visibilizadas dada la función social que ejercen. Una gran parte de la sociedad nicaragüense aún desconocen la existencia de estos tipos de universidades y, tampoco, las diferencias entre las universidades convencionales de las comunitarias e interculturales. Al respecto e inclusive, la Ley General de Educación de Nicaragua, Ley 582, aun cuando así las reconoce, no establece concepto alguno referidas a este tipo de universidades.

Algunos criterios diferenciadores de las universidades comunitarias e interculturales, de otras tradicionales son: patrimonio de derecho público sin fines de lucro; currículos integradores del conocimiento y saberes locales y pertinentes con la cosmovisión de las comunidades en su entorno; ubicación geográfica en áreas o regiones no atendidas por otras IES; promueven su acceso a sectores poblacionales históricamente marginados, y una amplia participación y gestión de actores locales claves en su gestación y/o fundación.

Adicionalmente, el carácter de lo público en su oferta académica está orientado más a intereses nacionales, al igual que su patrimonio. En cuanto a lo comunitario se refiere más al entorno geográfico inmediato a la ubicación del recinto respecto a las comunidades, y las carreras que ofertan priorizan el desarrollo de éstas. En tal sentido, aquí radica la importancia de la municipalización o regionalización de subsedes universitarias.

A nuestro juicio, la construcción del concepto de Universidad "Comunitaria $e$ Intercultural" se hace necesario a fin de diferenciarlas de las otras universidades tradicionales. En consecuencia, la Universidad Comunitaria e Intercultural es "Una Institución de Derecho público sin fines de lucro, de patrimonio Comunitario, organizada y constituida participativamente por diferentes actores que, mediante el diálogo de saberes y la integración de prácticas y conocimientos ancestrales responden prioritariamente tanto a escala temporal como espacial al desarrollo socioeconómico y cultural cosmovisionario de los Pueblos y Comunidades de su entorno". Sin entrar en debate si en el Modelo Educativo en las instituciones de Educación Superior (IES) en la Costa Caribe, lo comunitario implica lo intercultural o viceversa, y aun cuando el marco jurídico nacional e internacional sea favorable la implementación y consolidación de una universidades Comunitarias e Interculturales, esto implica un gran reto. 


\section{REFERENCIAS BIBLIOGRÁFICAS}

Alsina, M.R. (2001). La Interculturalidad en la modernidad actual. CIDOB. Encuentro internacional sobre interculturalidad, Barcelona, España.

Banks J. (1993). Multicultural Education: Development, Dimensions, and Challenges. Downloaded on, /12/Feb/2017 (pp. 2-8)

https://education.uw.edu/sites/default/files/20405019.pdf.

Bluefields Indian \& Caribbean University (BICU). (2017). Apuntes sobre la historia de la Universidad Comunitaria e Intercultural, BICU 1991-2017. Bluefields, R.A.A.C.S, Nicaragua.

Cabrero, F. (2008). Diversidad, pluralismo e interculturalidad. Editor Escuela Virtual, PNUD. Disponible en www.escuelapnud.org

Carratalá, A. (2012). Renovación metodológica de Teorías de la Comunicación ante el EEES. Vivat Academia, (117E), 247-266. Disponible en http://www.ucm.es/info/vivataca/numeros/n117E/DATOSS.htm\#12

Casillas M de L. M., Santini V. L. (2009). Universidad Intercultural Modelo Educativo. SECRETARÍA DE EDUCACIÓN PÚBLICA. Segunda Edición 2009. ISBN: 970-814206-9. Segunda edición. México.

Castillo Rodríguez N. y Ruiz López A. (2016). "Diversidad Social y cultural: trasformaciones y continuidades". En Conocimiento, crítica e intervención (pp. 92-93). Memorias del 5 Congreso Nacional de Ciencias Sociales.

Fornet R. B. (2007). Reflexiones de Raúl Fornet Betancourt sobre el concepto de interculturalidad. Consorcio Intercultural. Segunda edición, México.

García, F. (2000). Sociedad multicultural e interculturalismo versus inmigración extranjera: aportaciones teóricas para el debate. Disponible en https://scholar.google.es/scholar?hl=es\&as_sdt=0\%2C5\&q=García+2000+educación+m ulticultural+\&btnG

Gobierno de la República de Nicaragua. La Gaceta, Diario Oficial No 32 del 18 de febrero del 2016. Sumario. Texto de la Constitución Política de la Republica de Nicaragua con sus reformas incorporadas.

Gobierno de la República de Nicaragua. "Ley General de Educación. Ley No 582", Aprobada el 22 de marzo del 2006 Publicado en La Gaceta, Diario Oficial No. 150 del 03 de agosto del 2006.

Gobierno de la República de Nicaragua. "Reglamento a la Ley 28: Estatuto de Autonomía de las Regiones de la Costa Atlántica de Nicaragua". La Gaceta, Diario Oficial № 186 del 2 de octubre del 2003. .

Gómez M.G. (2009). "Competencias interculturales en instructores comunitarios que brindan servicio a la población indígena del estado de Chiapas". Revista Electrónica de Investigación Educativa, 12(1).

Gómez-Buendía, H., Arciniegas, E., Hernández, A. (2008). La democracia Moderna: Definición y Relación con la Multiculturalidad. Editor Escuela Virtual, PNUD. www.escuelapnud.org.

González, P. M. (1997). Gobiernos pluriétnicos: la constitución de las regiones autónomas en Nicaragua, $1^{\text {a }}$ edic. ed. Plaza y Valdés, México, D..F. pp 23-42.

Moreno T., Espinoza M., Solano E., Fresan M., (2016). "Evaluación de un Modelo 
Educativo Universitario: Una Perspectiva desde los Actores". En Revista Iberoamericana de Evaluación Educativa. Disponible en https://revistas.uam.es/index.php/riee/article/view/6655.

Näslund-Hadley, E., Meza, D., Arcia, G., Rápalo, R., \& Rondón, C. (2012). Educación en Nicaragua: Retos y Oportunidades. BID Banco Interamericano de Desarrollo, (IDB-TN458), 32. Disponible en http://www.iadb.org

Netto M. A. (2008). "Universidades comunitarias brasileñas: Un modelo para interiorizar la educación superior". Universidades, vol. LVIII, núm. 37, abril-junio, 2008, pp.3-48.

Organización Internacional del Trabajo (OIT). (1989). Convenio 169 de la Organización Internacional del Trabajo sobre Pueblos Indígenas y Tribales en Países Independientes, Oficina del Alto Comisionado para los Derechos Humanos. Disponible en www.unhchr.ch/spanish/html/menu3/b/62_sp.htm

Pedroza, M. E. (2015). El Modelo de gestión de la investigación, modelo $I+D+i$ de la UNAN Managua $\left(1^{\circ}\right)$. Managua: Universidad Nacional Autónoma de Nicaragua, Managua (UNAN-Managua) p.3.

Programa de Formación Intercultural Bilingüe para los Países Andinos (Proeib Andes)", Boletín, 2006. Disponible en http://cgi.proeibandes.org/.

Programa de las Naciones Unidas para el Desarrollo (PNUD). (2005). Informe de Desarrollo Humano 2005. Las Regiones Autónomas de la Costa Caribe. ¿Nicaragua Asume su Diversidad?, PNUD, Managua.

Rojas Cortez A. (2016). "Diversidad Social y cultural: trasformaciones y continuidades". En Educación superior intercultural y comunitaria. Dimensiones actorales $e$ institucionales en Mexico y Oaxaca (pp.184-185). Memorias del 5 Congreso Nacional de Ciencias Sociales.

SEP. Secretaria de Eduacion Publica (2017). Modelo Educativo. Disponible en https://es.slideshare.net/hugomedina36/modelo-educativo-2017.

Sandoval E.A., Guerra G.E. (2007). "La interculturalidad en la educación superior en México" Ra Ximhai, mayo-agosto, año/Vol. 3, Número 2 Universidad Autónoma Indígena de México Mochicahui, El Fuerte, Sinaloa. pp. 273-288

Tubino F., Mansilla K. (2012). UNIVERSIDAD E INTERCULTURALIDAD Desafíos para América Latina. 1era Edic. Pontificia Universidad Católica del Perú Av. Universitaria 1801, San Miguel, Lima 32. (pp. 55-60).

Tünnerman, B. C. (2008) Modelos Educativos y Académicos. Disponible en http://www.enriquebolanos.org/media/publicacion/Modelos\%20educativos\%20y\%20aca demicos.pdf

Universidad Nacional Autónoma de Nicaragua - Managua (UNAN-Managua). (2018). El Nuevo Modelo Educativo de la UNAN- Managua [Archivo de video]. Disponible en https://www.youtube.com/watch?v=-eg4Ac7nDjo\&feature=youtu.be

Universidad de las Regiones Autónomas de la Costa Caribe Nicaragüense (URACCAN). (2016). Proyecto Educativo Institucional (PEI). Nueva Guinea, Región Autonoma Costa Caribe Sur, Nicaragua-.

Walsh, C. (2009). "Interculturalidad crítica y pedagogía de-colonial: apuestas desde el insurgir, re-existir y re-vivir". Revista educação intercultural hoje en América Latina, La Paz, Bolívia (pp 8-9). 